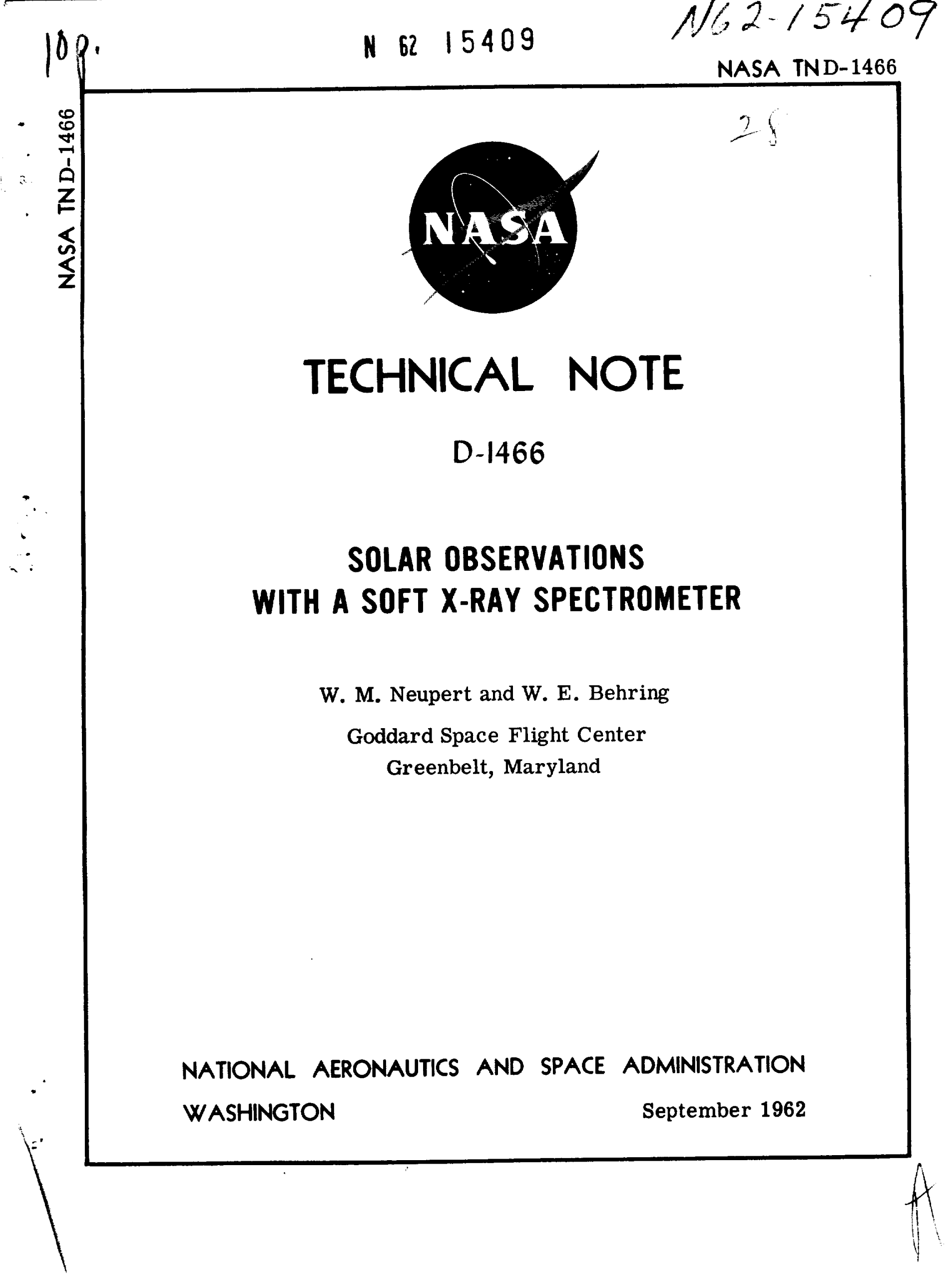




\section{SOLAR OBSERVATIONS WITH A SOFT X-RAY SPECTROMETER}

by

W. M. Neupert and W. E. Behring

Goddard Space Flight Center

SUMMARY

A grazing-incidence soft $\mathrm{x}$-ray spectrometer designed for long term operation in a satellite has been used for preliminary observation of the solar soft x-ray spectrum during an Aerobee rocket flight. The instrument employs as its dispersing element a lightly ruled concave glass grating with a radius of curvature of 1 meter. The resultant spectrum is scanned with a Bendix open-window photomultiplier mounted behind the exit slit on a carriage that moves the assembly along the Rowland circle from $10 \mathrm{~A}$ to $400 \mathrm{~A}$. Wavelength calibration is obtained through observation of the carbon $\mathrm{K}$ emission band in the laboratory together with observation during flight of the He II Lyman-alpha line. A study of the reflecting power of the grating has been made which, together with an investigation of the efficiency of the detector now being planned, will provide an estimate of the instrumental efficiency at one or more wavelengths. Observations during a recent rocket flight indicate the presence of numerous emission lines in the $120-400 \mathrm{~A}$ region. Tentative identification of lines produced by highly ionized atoms has been made. 



\section{CONTENTS}

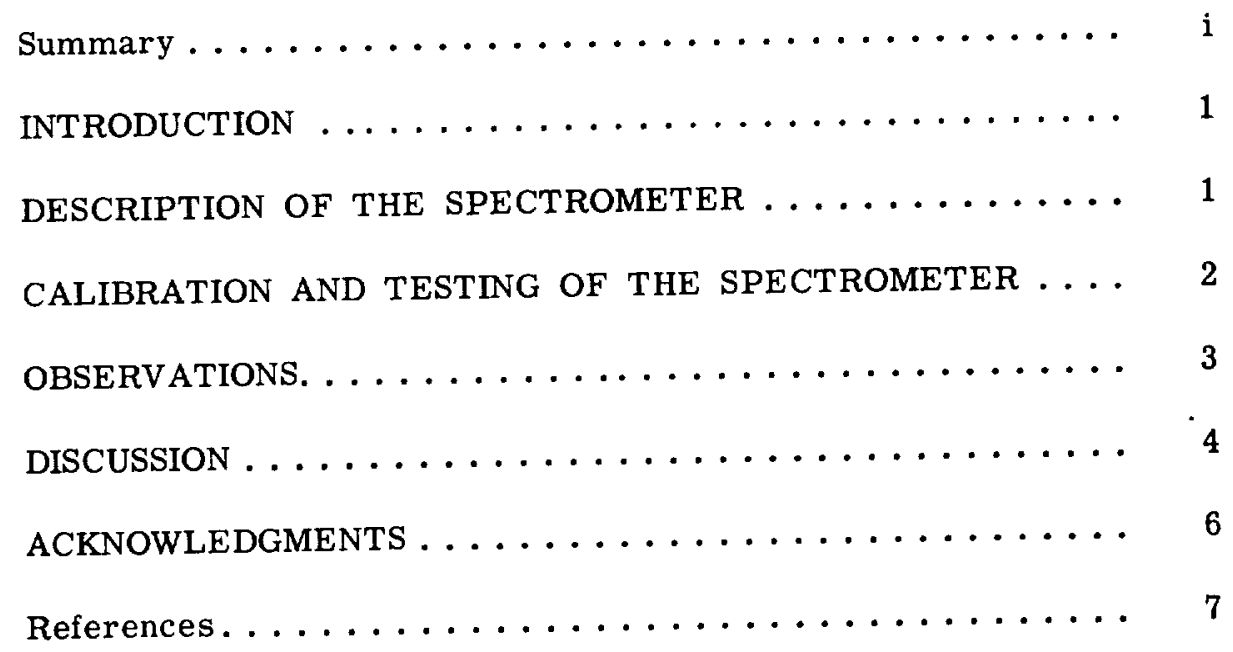




\title{
SOLAR OBSERVATIONS WITH A SOFT X-RAY SPECTROMETER
}

\author{
by \\ W. M. Neupert and W. E. Behring \\ Goddard Space Flight Center
}

\section{INTRODUCTION}

Since 1959, studies of the far ultraviolet solar spectrum have been made with grazingincidence spectrographs and spectrometers carried above the atmosphere by rockets and pointed at the sun by means of a biaxial pointing control developed at the University of Colorado. These observations have yielded a wealth of information about the radiations which cause ionization in the upper layers of the atmosphere. Violett and Rense ${ }^{1}$ at Colorado, Hinteregger ${ }^{2}$ at the Air Force Cambridge Research Center, and most recently Tousey ${ }^{3}$ and his co-workers at the Naval Research Laboratory, have succeeded in observing the Lyman-alpha line of ionized helium at $304 \mathrm{~A}$ and numerous other emission lines at shorter wavelengths.

This paper presents results obtained by a soft $\mathrm{x}$-ray spectrometer, designed primarily to make observations from a satellite, during a preliminary Aerobee rocket flight on September 30, 1961.

\section{DESCRIPTION OF THE SPECTROMETER}

During operation the spectrometer (Figure 1) is pointed at the center of the solar disk with an accuracy of several minutes of arc. In this orientation, radiation from the entire solar disk and inner corona passes through the entrance slit and strikes a concave grating mounted in grazing incidence, the angle of incidence being 88 degrees. The grating, an original which has been ruled in a special glass by the Nobel Institute in Stockholm, has 576 lines per millimeter on a blank whose radius of curvature is 1 meter. The exit slit and detector are mounted on a carriage which is driven on a circular rail so that the exit slit scans along the Rowland circle from 10 to $400 \mathrm{~A}$. The plane of the exit slit stays approximately perpendicular to the diffracted ray at all positions along the track, thus keeping the spectral passband nearly constant for all angles of diffraction. The 50-micron entrance and exit slits provide a spectral passband of $1.7 \mathrm{~A}$ and permit resolution of lines $0.85 \mathrm{~A}$ apart. The detector is a windowless photomultiplier developed by the Bendix Corporation 


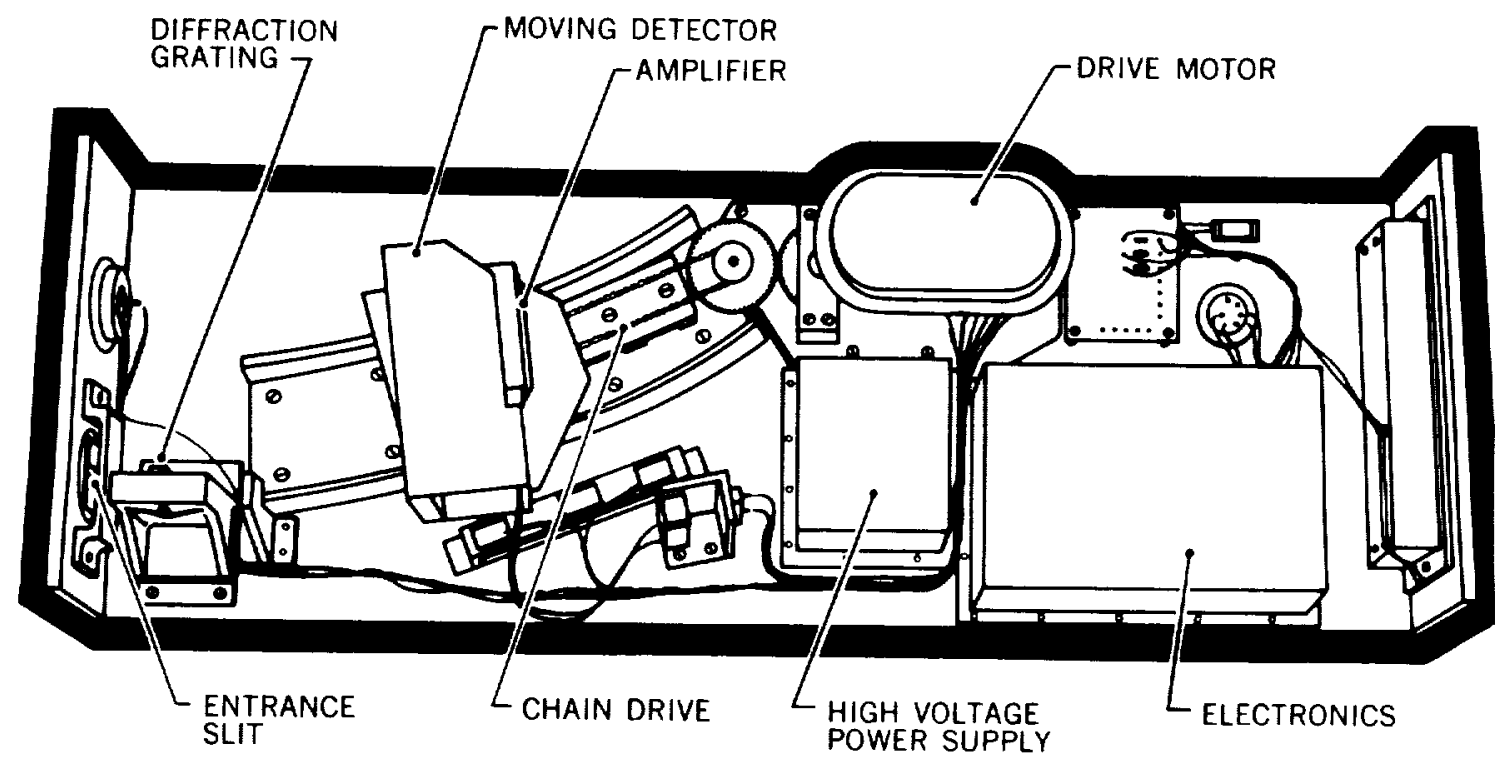

Figure 1-Top view of the soft $x$-ray spectrometer showing placement of the major components of the instrument

specifically for use in this spectrometer and now commercially known as the M306. A tungsten photocathode was chosen to minimize response to wavelengths above $1500 \mathrm{~A}$.

A detailed description of the instrument has been presented elsewhere; only an abstract has been published ${ }^{4}$, but publication of the complete document is planned.

\section{CALIBRATION AND TESTING OF THE SPECTROMETER}

Because the instrument's range includes only one order of the only emission line whose wavelength is known with accuracy - the Lyman-alpha line of ionized helium - it is impossible to obtain wavelengths by the usual method of relating, through overlapping orders, lines of unknown wavelength to known lines of well established wavelength. Rather wavelengths were obtained by means of the grating equation, with values for the grating constant and the angle of incidence computed by using the observed positions on the Rowland circle at which radiations of two different wavelengths were focussed. The radiations chosen were the carbon $\mathrm{K}$ emission band at $44 \mathrm{~A}$, which has identifiable features and can easily be generated in the laboratory, and the He II Lyman-alpha line at $304 \mathrm{~A}$, which is observed in the sun. Lack of coincidence of the Rowland circle with the curve generated by the moving exit slit, nonlinearities in the driving linkage, and variations in motor speed were taken into account to yield a more accurate calibration than could be obtained by inserting nominal instrumental parameters into the grating equation. The wavelength scale 
was checked by calculating the position of the carbon band in the second order. Agreement to within $0.3 \mathrm{~A}$ was obtained.

To observe the existence of continua in the spectrum at short wivelengths, it was necessary to determine the response of the instrument to scattered radiation, which was expected to be primarily hydrogen Lyman-alpha radiation. This measurement was carried out with a closed ( $\mathrm{LiF}$ window) hydrogen discharge tube at a distance great enough to approximate the angle subtended by the sun under actual observing conditions. The response to charged particles was also studied and minimized by covering the entrance slit with a screen operated at a positive potential of 18 volts. The photocathode of the detector was held at a large negative voltage to reduce its susceptibility to electrons.

A program is currently underway for comparing the Bendix photomultiplier with proportional and Geiger counters of known sensitivity at selected wavelengths from $44 \mathrm{~A}$ to 304A. Information obtained in these studies, combined with values of grating reflectivity in first and higher orders obtained by Prof. D. H. Tomboulian of Cornell University, will provide the basis for estimates of absolute fluxes at discrete wavelengths in the solar spectrum.

\section{OBSERVATIONS}

Figure 2 shows a scan through the solar spectrum taken near the rocket's maximum altitude. The telemetered data were processed and plotted by computer to obtain this
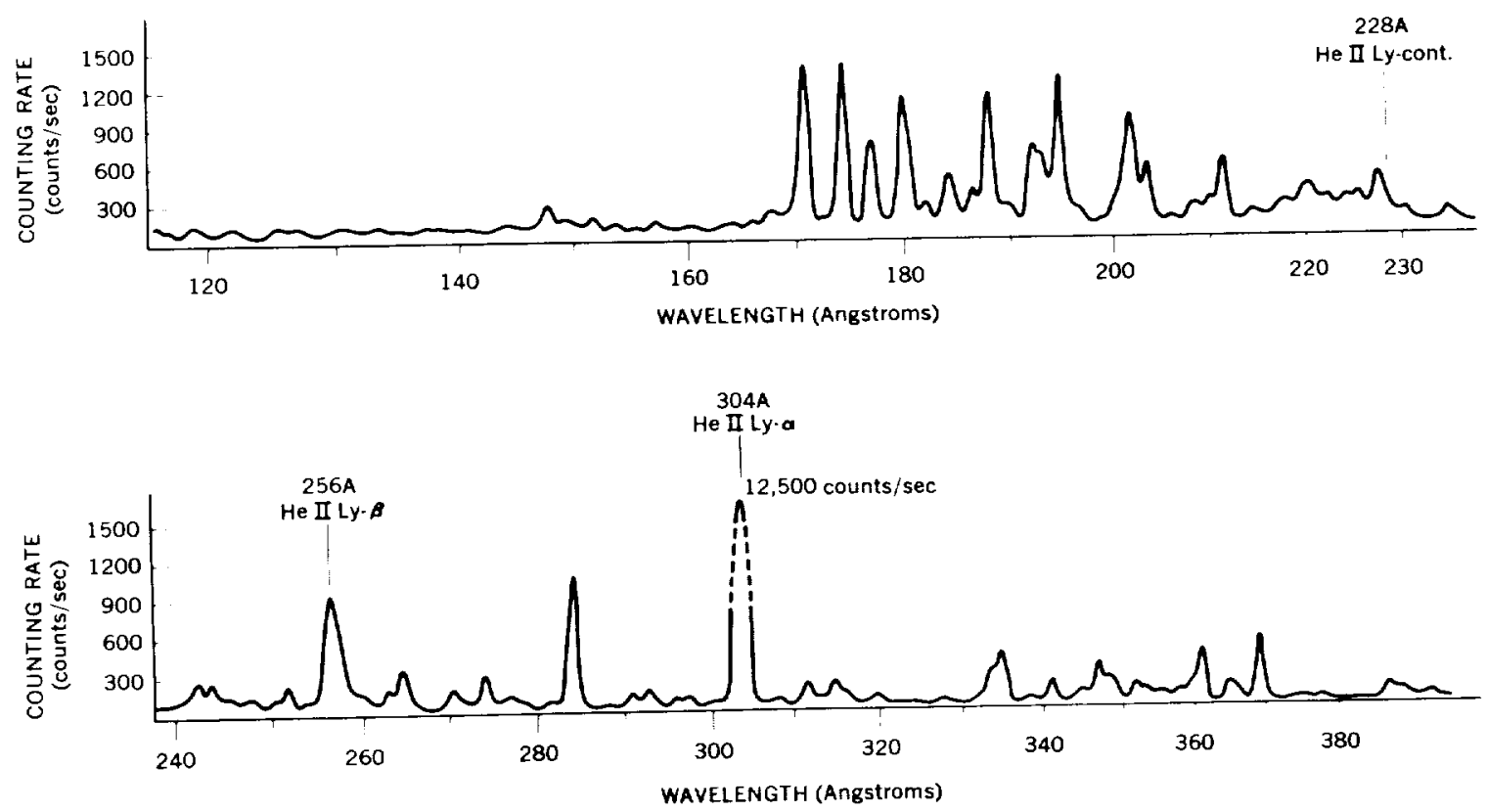

Figure 2-The solar spectrum from 120 to $400 \mathrm{~A}$ as recorded near peak altitude $(201-216 \mathrm{~km})$ during an Aerobee rocket flight at 1433 GMT on September 30, 1961 
curve. The wavelength regions from 120 to $170 \mathrm{~A}$ and 220 to $240 \mathrm{~A}$ represent the average of data taken in three different scans in an attempt to improve the reliability of faint lines and to provide continuity in the portions of the spectra originally containing position marker pulses. Comparison of average observed counting rates with laboratory scattered-light measurements indicates that the counting rate attributable to the first order soft $x$-ray spectrum is lost in the scattered light at wavelengths shorter than $60 \mathrm{~A}$. The region from 60 to $120 \mathrm{~A}$ has not been analyzed because of low counting rates and consequent poor statistics. Partial compensation for this can be made by combining many observations taken under similar solar conditions. Data from the spectrometer mounted in the first Orbiting Solar Observatory satellite (OSO-1) will be used for this purpose.

\section{DISCUSSION}

As in previous observations, the He II Lyman-alpha line is by far the most intense emission line below 400A. Another emission line, at 256A, which shows unresolved structure is partially attributable to He II Lyman-beta. Fainter lines also appear at wavelengths expected for several higher members of the Lyman series.

On the basis of rocket data alone, it is not possible to detect any continua. Neither a bremsstrahlung continuum, expected by Elwert ${ }^{5}$ to have a peak at $50 \mathrm{~A}$, nor recombination continua, expected to have series limits below $100 \mathrm{~A}$ for heavy elements and at $228 \mathrm{~A}$ for He II, can be identified.

An attempt has been made to extend to shorter wavelengths and heavier elements the work of Rense, Tousey, and Hinteregger in identifying resonance lines of highly ionized atoms. At longer wavelengths those authors have identified lines produced by several stages of ionization of $\mathrm{C}, \mathrm{N}$, and $\mathrm{O}$, and also by $\mathrm{Mg}, \mathrm{Si}, \mathrm{Ne}$, and $\mathrm{Fe}$. The extension of isoelectronic sequences to heavier elements leads to resonance lines with wavelengths below 400A. The tabulation of emissions compiled by Varsavsky ${ }^{6}$ was used in making tentative identifications. The criteria applied in making these assignments were:

1. Agreement with theoretically extrapolated values of the spacing and intensities of members of a multiplet assuming, for the intensities, an optically thin corona;

2. Approximate agreement, in wavelength, with theory for lines not yet observed under laboratory conditions;

3. Observation of more than one stage of ionization.

A preliminary analysis of the spectrum was made for ions of heavy elements which, by observation of forbidden lines found in the visible spectrum, are known to exist in the solar atmosphere. These are ions of $\mathrm{Fe}, \mathrm{Ni}$, and, with lesser abundances, $\mathrm{Ca}$ and $\mathrm{A}$. Identification of iron multiplets (Figure $3 \mathrm{a}$ ) on the basis of one observation is made difficult 


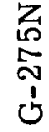
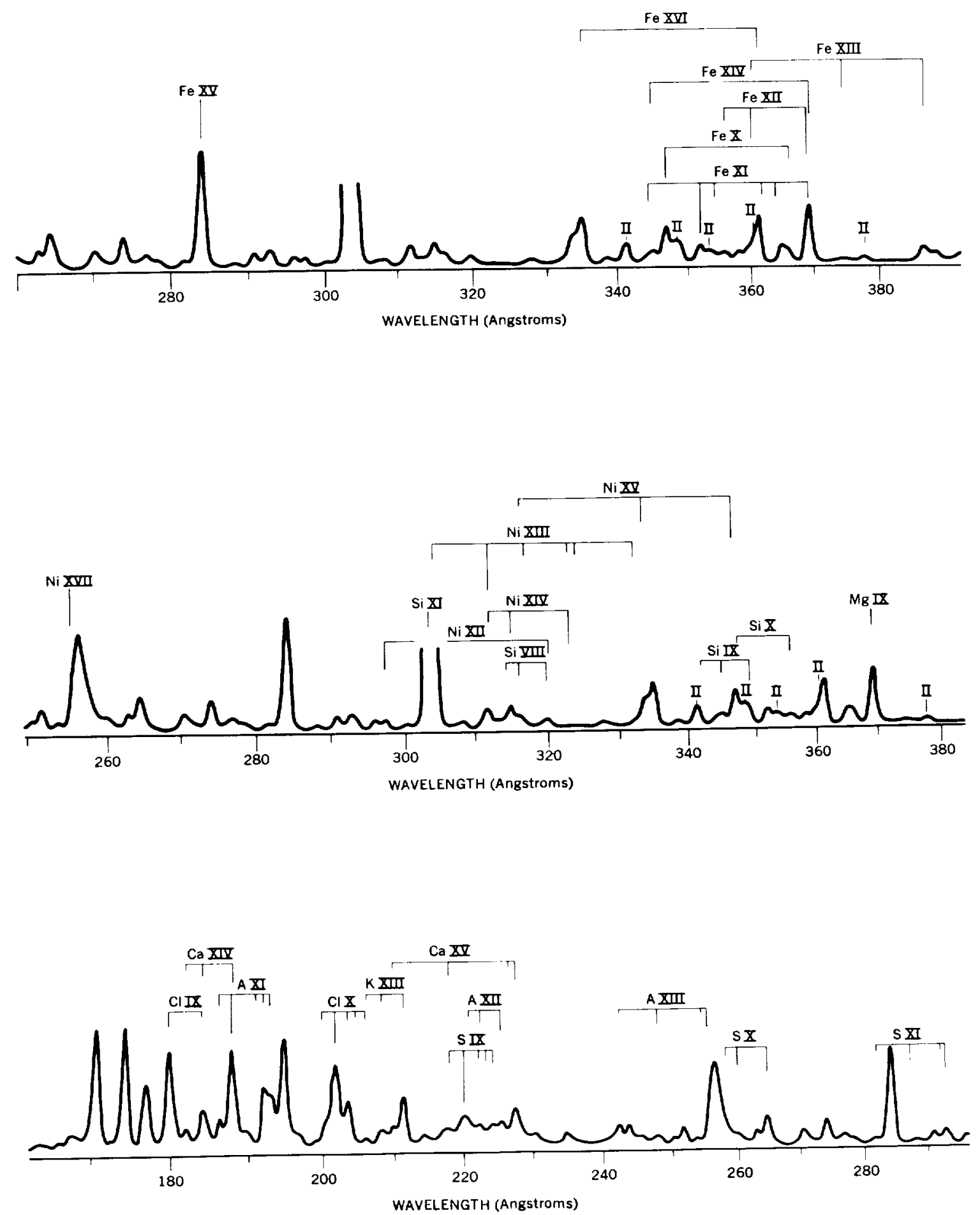

Figure 3-Tentative assignments of coronal emission lines in the region from 160A to 400A. The lengths of vertical marks denoting the positions of the lines are proportional to the calculated weighted oscillator strengths for the lines. 
by the presence of strong second order lines as well as by the superposition of the multiplets themselves. Only the Fe XV line has been calculated with accuracy; it has been identified by Edlen ${ }^{7}$ with a strong line at $284 \mathrm{~A}$. The coronal resonance lines of highly ionized nickel are expected at shorter wavelengths and with lower intensities than the iron lines (Figure $3 \mathrm{~b}$ ). Because of the decreasing grating efficiency with increasing wavelength in the region above $200 \mathrm{~A}$, the difference in intensity between $\mathrm{Ni}$ and Fe lines is probably greater than the data indicate. On the basis of these assignments it appears that the line at approximately 335A may be a blend of Ni XV and Fe XVI. Possible assignments for $\mathrm{Mg}$ and $\mathrm{Si}$ are also given in Figure $3 \mathrm{~b}$.

Figure 3c shows assignments made for $\mathrm{Ca}, \mathrm{Si}, \mathrm{Cl}$ and $\mathrm{K}$. Multiplets for each element are given in a horizontal line and multiplets belonging to the same isoelectronic sequence are found by following a diagonal. The resonance lines of $\mathrm{Ca}$ XII and $\mathrm{Ca}$ XII, two ions observed in the visible coronal spectrum, cannot definitely be associated with any of the emissions in the far ultraviolet spectrum. Neither is a correlation observed, although it is expected, for A X. The intensity of the multiplet assigned to potassium is not expected on the basis of the relative abundances of $\mathrm{Ca}$ and $\mathrm{K}$.

All of the foregoing assignments must be regarded as tentative and subject to modification upon analysis of $O S O-1$ observations which will permit additional criteria to be applied for the identification of multiplets. These further conditions are:

1. Constancy, with time, of the intensity ratios of members of a multiplet, assuming no changes in the opacity of the corona with time.

2. Regularity in the intensity variations over the observed staures of ionizations.

3. Where possible, agreement in the intensity variations for each stage of ionization with variations in the corresponding visible coronal lines.

Observations from OSO-1 will also permit upper limits to be placed on continua in the soft $x$-ray spectrum; they will also provide a means of comparing any enhancements in the spectrum during periods of solar activity with changes at $x$-ray wavelengths and with solar radio bursts observed on the earth.

\section{ACKNOWLEDGMENTS}

The authors wish to thank Professor D. H. Tomboulian of Cornell University for the benefit of his knowledge of instruments and phenomena in the soft $x$-ray region; and for his study of the gratings, on the basis of which the flight gratings were selected. We also wish to express our appreciation to Mr. Theodore P. Stecher of Goddard Space Flight Center for many valuable discussions concerning the interpretation of the spectrum. 


\section{REFERENCES}

1. Violett, T., and Rense, W. A., "Solar Emission Lines in the Extreme Ultraviolet," Astrophys. J. 130(3):954-960, November 1959

2. Hinteregger, H. E., Damon, K. R., and Hall, L. A., "Analysis of Photoelectrons from Solar Extreme Ultraviolet," J. Geophys. Res. 64(8):961-969, August 1959

3. Austin, W. E., Purcell, J. D., and Tousey, R., "Solar Spectrum Photographed to 168A at Grazing Incidence without Stray Light," J. Opt. Soc. Amer. 52(5); 597, May 1962 (Abstract)

4. Behring, W. E., Neupert, W. M., and Nichols, W. A., "Far Ultraviolet Spectrometer for Solar Observations," J. Opt. Soc. Amer. 52(5):597, May 1962 (Abstract)

5. Elwert, G., "Theory of X-Ray Emission of the Sun," J. Geophys. Res. 66(2):391-401, February 1961

6. Varsavsky, C. M., "Some Atomic Parameters for Ultraviolet Lines," Astrophys. J. Suppl. 6(53):75-107, March 1961

7. Firor, J., and Zirin, H., "Observations of Five Ionization Stages of Iron in the Solar Corona," Astrophys. J. 135(1):122-137, January 1962 